\title{
A Quad-band Compact Diversity Antenna for Mobile Handset Devices
}

\author{
Yang Yang ${ }^{1} * *$, Yuan'An $\mathrm{Liu}^{2}$ and Fan $\mathrm{Wu}^{3}$ \\ Beijing University of Posts and Telecommunications, Beijing, China \\ I**yangyang@bupt.edu.cn, ${ }^{2} y u l i u @ b u p t . e d u . c n,{ }^{3}$ wufanwww@bupt.edu.cn
}

\begin{abstract}
A quad-band planar compact diversity antenna for mobile handset devices is presented in this paper. The antenna unit of the proposed antenna is a "paperclip" structure consisted by an inverted L-shaped strip and a $C$-shape meander branch connected by a metallic via hole. The radiation structure of the proposed antenna is compact, $15.6 \times 25 \mathrm{~mm}^{2}$. Simulated and measured results show that the proposed antenna can operate at 0.915/2.45/3.5/5.8GHz WSNs, WLAN and LTE bands with quasi-monopole radiation patterns. Two diversity antenna geometry are compared and discussed in the overall size of $60 \mathrm{~mm} \times 100 \mathrm{~mm}$ substrate similar like the size of modern mobile handset devices. A low Enveloped Correlation Coefficient $(E C C)$ characteristic are achieved by the optimized diversity design. Together with compact volume, the proposed diversity antenna is a good candidate for mobile handset device applications.
\end{abstract}

Keywords: Handsets, Quad-bands, Diversity Antenna, Compact

\section{Introduction}

Mobile handset devices such as cellphone, tablet computer combine many system communication technologies together. For the rapid evolutions of mobile communication technology, the Long Term Evolution (LTE) and LTE-advanced take the MIMO technology to achieve higher channel capacity. However, the restrict space limitation in the mobile handset devices design increases the difficulties of multi-band antenna toward compact, low profile and board bandwidth for the devices. Therefore, a deals of research and approach has been achieving to increase bandwidth and reduce the antenna volume [1-5]. Slot structure was used to achieve a multi-band resonance with a small size in some studies [1-3]. In [2], An instance is shown that a printed planar antenna with a size of $30 \times 30 \mathrm{~mm} 2$ generated three resonant modes by two dissimilar inverted L-shaped slots and a mushroom-shaped slot loaded into the antenna. Also, some other promising structures have been proposed, such as monopole [4, 5]. In the diversity aspect, some efforts are made to enhance the diversity performance and reduce the volume. In [7], the UWB technology was introduced. The antenna consists of two planar-monopole (PM) antenna $(26 \mathrm{~mm} * 40 \mathrm{~mm})$ elements with microstrip-fed printed on one side of the substrate and placed perpendicularly to each other to achieve good isolation. In [8], the researchers designed the diversity PIFAs $(31.5 \mathrm{~mm} * 9 \mathrm{~mm})$ loaded with a series combination of an inductor and a varactor to simultaneously achieve miniaturization and tunability. Although the aforementioned approaches on multiband antenna made efforts in size and multiple resonant band, however, all of them are unable to provide $0.9 \mathrm{GHz}$ operating frequency band and multiband ability at same time which is critical for handset devices application.

In this paper, a compact planar multi-band antenna unit with radiation elements size of $15.9 \times 25 \mathrm{~mm} 2$ is proposed and two types of different diversity array are also proposed and discussed. The antenna can cover WSNs $915 / 2400 \mathrm{MHz}$, LTE 2100/2300/3500MHz, WLAN $2400 / 5800 \mathrm{MHz}$ and GSM 1700/1800/1900MHz. The whole designed is simulated 
using CST Microwave Studio and fabricated. The simulated and measured results are presented and are in good agreement. The rest of the paper is organized as follows. Section 2 presents antenna design both of the antenna unit and diversity design. Section 3 gives parametric study. The current distributions are analyzed in single antenna scenario and diversity scenario in Section 4. Section 5 shows the simulated and measured results. Conclusion is given in Section 6.

\section{Antenna Design}

\subsection{Single Antenna Design}

The concept of quad-band monopole antenna evolves from the simple inverted $-\mathrm{L}$ shape antenna, as shown in the left prototype antenna in Figure 1. To acquire a low resonance mode, the second prototype (middle antenna in Figure 1) is introduced in the design. Due to the longer folding strip and the shorter strip of the bottom side, the ANT 2 generates three resonant frequencies at $1.22,3.30$ and $5.31 \mathrm{GHz}$, while only the last two modes excited successfully. By combining two prototypes together and optimizing the geometry parameters, the proposed monopole antenna unit generates four different resonate modes respectively.

The quad-band antenna unit is designed as a quarter- wavelength antenna, which means that the length of each resonant branch should follow the antenna electric length equation (1).

$$
\lambda_{g}=\frac{c}{f \sqrt{\varepsilon_{f f}}}, \varepsilon_{f f}=\frac{\varepsilon_{r}+1}{2}
$$

In (1), $\mathrm{c}$ is the speed of light, $\mathrm{f}$ is the desired resonant frequency, $\varepsilon_{\mathrm{ff}}$ is the effective relative permittivity. The length of resonant branch is $\approx \lambda \mathrm{g} / 4$. The geometry of the proposed monopole antenna is shown in Figure 2. The antenna was designed on a low cost, durable FR4 substrate with relative dielectric constant $\varepsilon_{\mathrm{r}}=4.4$, loss tangent $\tan \delta=$ 0.02 . A $50 \Omega$ coaxial cable with a SMA connector was used to feed the antenna. The front radiation element on the top layer is an Inverted-L strip with a shorter vertical strip added at the end. A paperclip-like radiation structure is on the bottom layer of the antenna, and connects with the Inverted-L strip by a metallic via hole at the end of L1. The papercliplike structure consist by two branches, a longer meander branch and a shorter branch. A rectangular parasitic tuning block is introduced near the feed to improve the resonant characteristics of high frequency band by optimizing the size parameters.

The geometry of the antenna is carefully designed and optimized, the total length of Lshape strip is $25.5 \mathrm{~mm}(\mathrm{~L} 1+\mathrm{L} 2+\mathrm{W} 1 \mathrm{~mm})$, the longer folding strip of the "paperclip" is $65.68 \mathrm{~mm}(\mathrm{~L} 1+1+6+6.5+21.2+3.2+\mathrm{L} 3+11.68 \mathrm{~mm})$ and the shorter arm of paperclip-like structure is $14.1 \mathrm{~mm}(\mathrm{~L} 1+1+5.1 \mathrm{~mm})$. Other parameters list as followed, $\mathrm{L} 1=8 \mathrm{~mm}, \mathrm{~L} 2=$ $4 \mathrm{~mm}$ and $\mathrm{L} 3=8 \mathrm{~mm}$.

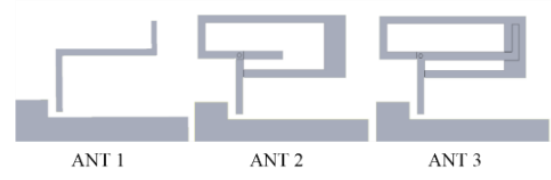

Figure 1. Different Prototypes Antenna

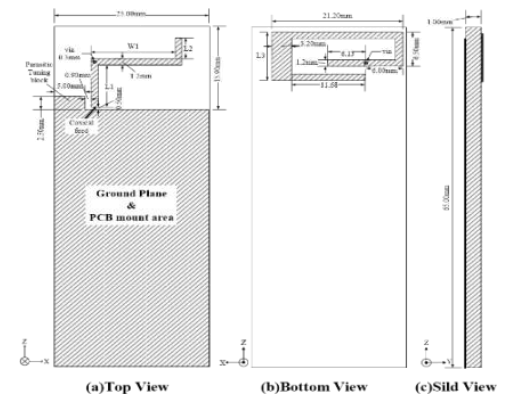

Figure 2. Geometry of Proposed Antenna 
The difference of prototypes and proposed antenna unit in impedance resonance characteristic are shown in Figure 3. As depicted in Ant 1 provide only one resonant mode center at $2.57 \mathrm{GHz}(1.96-2.77 \mathrm{GHz})$. Due to the longer folding strip and the shorter strip of the bottom side, the ANT 2 generates three resonant frequencies at 1.22, 3.30 and $5.31 \mathrm{GHz}$, while only the last two modes excited successfully. the proposed monopole antenna finally obtained a four band resonance $(\mathrm{S} 11 \leq-10 \mathrm{~dB})$, the first frequency band $0.90-0.94 \mathrm{MHz}$, the second frequency band $1.69-2.59 \mathrm{GHz}$, the third frequency band 3.42$3.61 \mathrm{GHz}$, and the fourth frequency band $5.50-5.89 \mathrm{GHz}$ respectively.

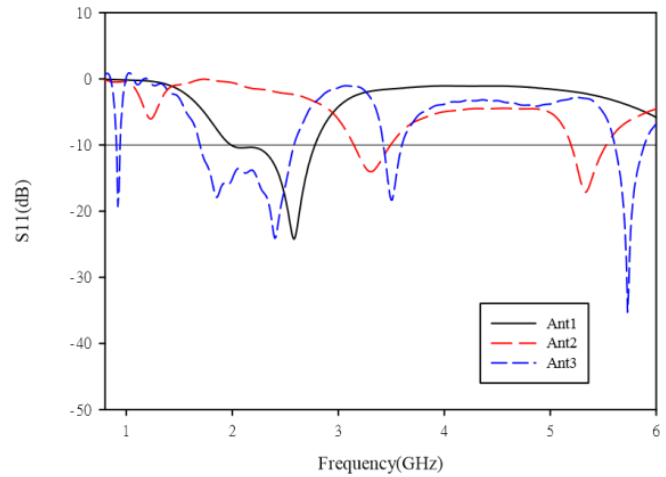

Figure 3. Corresponding Return Loss Characteristics

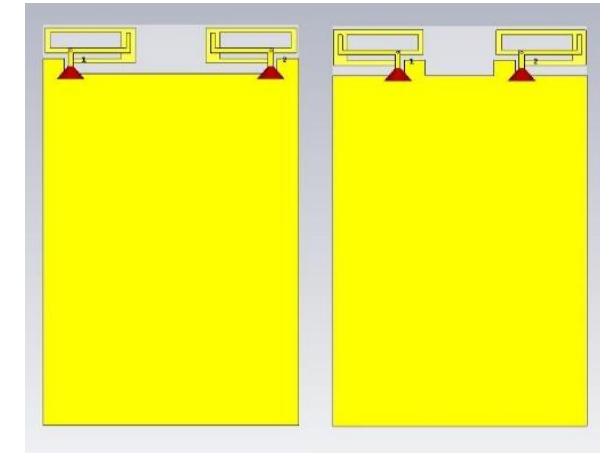

Figure 3. Two Types of Diversity Geometry Type A(left) \& Type $B$ (right)

\subsection{Diversity Antenna Design}

The diversity antenna arrays are designed in the size of $60 \mathrm{~mm} * 100 \mathrm{~mm}$ FR-4 substrate. To reduce the complexity of design, the arrays take the simple space diversity techniques. Two different geometry are introduced, shown in Figure 3. Type-A array is "front to front" geometry and Type-B takes "back to back" geometry design.Fig.4 shows the characteristics of transmission characteristics of the two diversity design. It can observe that the resonance shifts to lower frequency at higher resonant mode (from $5.7 \mathrm{GHz}$ down to $5.5 \mathrm{Ghz}$ ) and a little shift to higher frequency at $3.5 \mathrm{Ghz}$, both in Type-A and Type-B. However, the Type-A has more advantage at impedance bandwidth (at $2.4 \mathrm{Ghz}$ band and $3.5 \mathrm{GHzband}$ ) and high port isolation (2.4Ghz band). Therefore, the Envelop Correlational Coefficient (ECC) of the Type-A is simulated and calculated, shown in Fig.5.It depicts that the proposed diversity antenna has good independency between the two antenna unit at all operational frequency band.

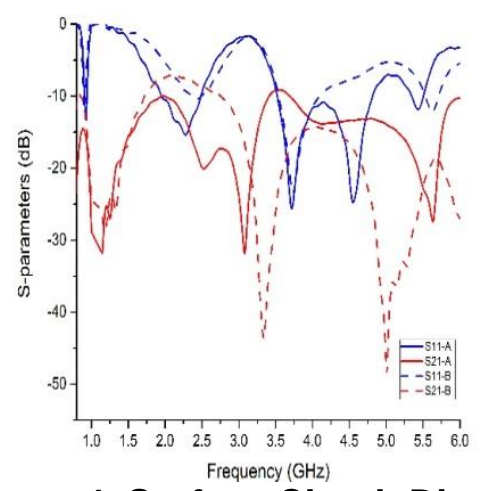

Figure 4. Surface Circuit Distribution at Different Frequency

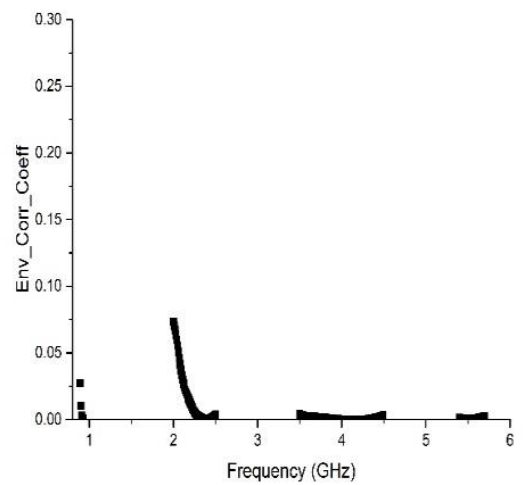

Figure 5. Envelop Correlational Coefficient of Proposed Diversity Antenna(Type-A) 


\section{Parametric Study}

More antenna characteristics can be learned by changing the dimensional parameters. Several specific parameters of the proposed antenna are investigated.

Return loss characteristics changes when the distance between the radiation structure and the ground plane (L1). In Figure 5, it can be noticed that the first resonant point is barely affected with the changes, while other three resonant point present move to higher frequency and the impedance matching improved when L1 decreases from $9 \mathrm{~mm}$ to $7 \mathrm{~mm}$. The $-10 \mathrm{~dB}$ impedance bandwidth of second and fourth resonant band reach its max value when L1 is $8 \mathrm{~mm}$. Effect of changing L2 on reflation characteristics are demonstrates in Figure 6. It implies that the changes affect the resonant mode of all the resonant band. When L2 gets longer, all resonant frequencies shift to lower frequencies. The impendence matching at $2.1 \mathrm{GHz}$ improves when $\mathrm{L} 2$ reaches to $4 \mathrm{~mm}$, while deteriorates when $\mathrm{L} 2$ is $6 \mathrm{~mm}$.

In Figure 7 depicts the variation of S11 for different values of L3. Note impendence matching improve at $3.5 \mathrm{GHz}$ and degrade at second resonant band with distinct bandwidth shirking when L3 increase from $7 \mathrm{~mm}$ to $9 \mathrm{~mm}$. Frequency shifting occur at the first and the fourth resonant mode with L3 varying as well. Figure 8 displays the S11 at different W1. It can be observed that as W1 increase, the return loss of $2.1 \mathrm{GHz}$ and the third frequency band improved, while mismatching occurs at $2.45 \mathrm{GHz}$.

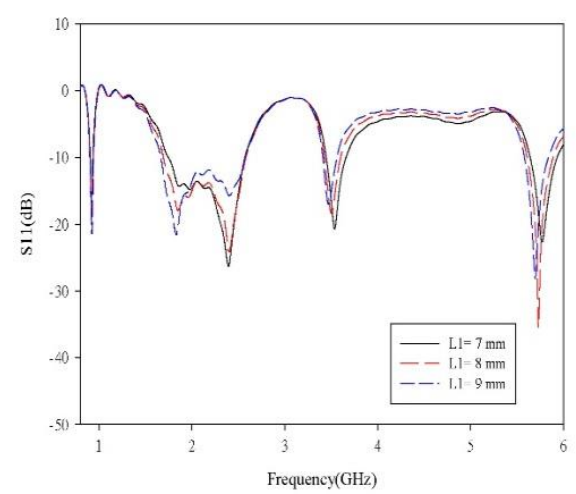

Figure 6. Return Loss against Frequency at Various L1

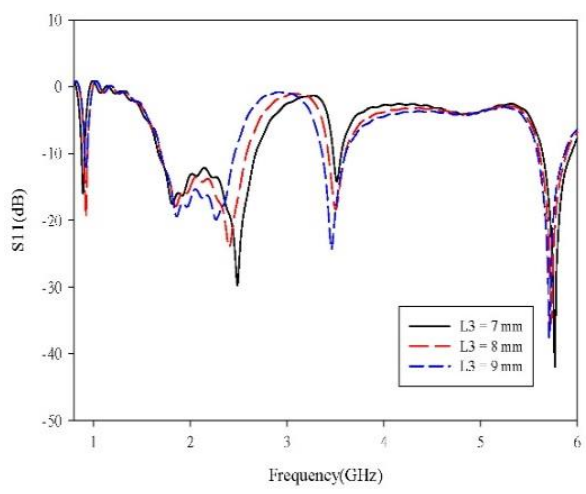

Figure 7. Return Loss against Frequency at Various L3

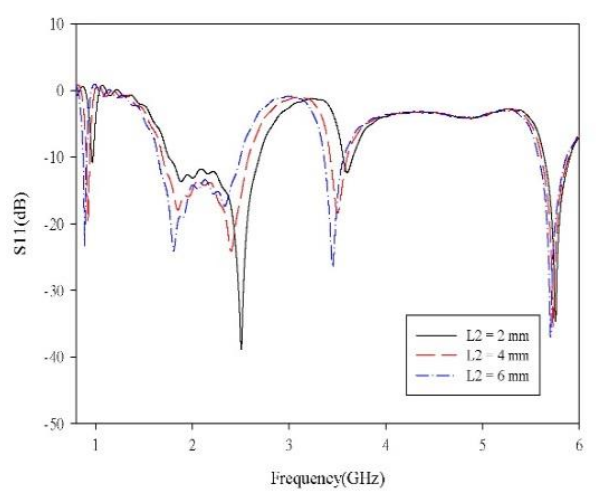

Figure 6. Return Loss against Frequency at Various L2

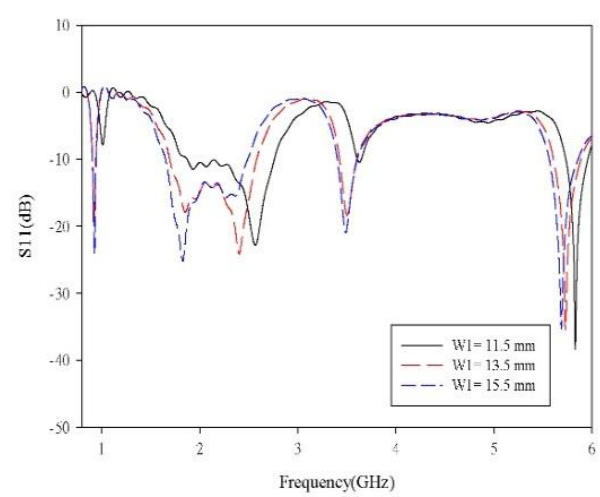

Figure 8. Return Loss against Frequency at Various W1 


\section{Current Distribution}

\subsection{Single Antenna Scenario}

To investigate the radiation mechanism in single antenna scenario, the current distribution at different resonances are simulated by CST Microwave Studio, shown in Figure 9.The strong current can be observed mainly at the upper strip of paperclip-like strip in Figure 9(a), and it indicates that upper part of the "paperclip" element generate resonance at $0.915 \mathrm{GHz}$. For $2.45 \mathrm{GHz}$ and $3.5 \mathrm{GHz}$ operation, in Figure $9(\mathrm{~b})$, the strong current flows mainly along the L-shape strip and the lower part of the meander strip. Likewise, the result at $5.7 \mathrm{GHz}$ in Figure 9 (d) is caused by the shorter strip on the bottom layer and a part of front radiation structure.

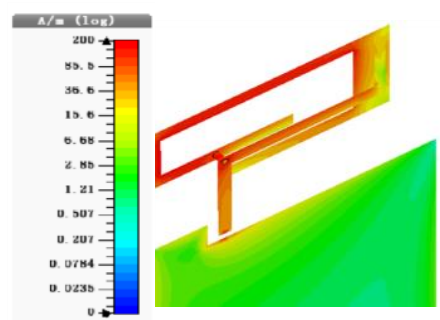

(a) $0.915 \mathrm{GHz}$

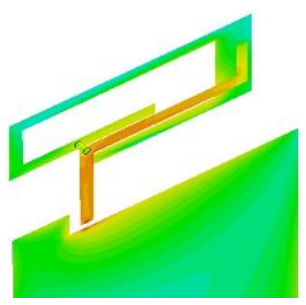

(b) $2.45 \mathrm{GHz}$

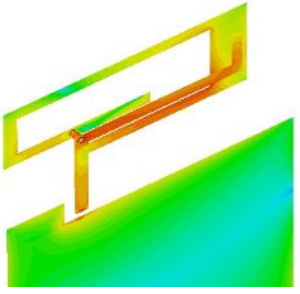

(c) $3.5 \mathrm{GHz}$

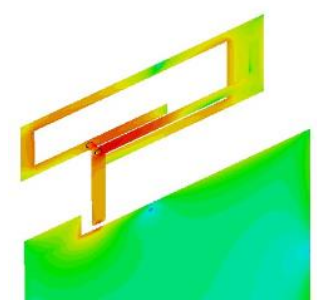

(d) $5.7 \mathrm{GHz}$

Figure 9. Surface Circuit Distribution at Different Frequency

\subsection{Diversity Antenna Scenario}

The proposed diversity antenna takes the space diversity technique and two antenna array geometry are simulated. In the diversity antenna scenario, the port isolation performance is highly relative with the current distribution between two feed port. Figure 10 and Figure 11 show the surface circuit distribution at specific $2.45 \mathrm{GHz}$ and $5.5 \mathrm{GHz}$ of Type-A and Type-B antenna array. In Figure 10, at $2.45 \mathrm{GHz}$ operation, more leak current occurs at Type-B than that in Type-A. Likewise, at $5.5 \mathrm{GHz}$, Type-A also has less surface current between two feed port. This phenomenon explains the result of the return loss characteristics of two antenna port in Figure 4.
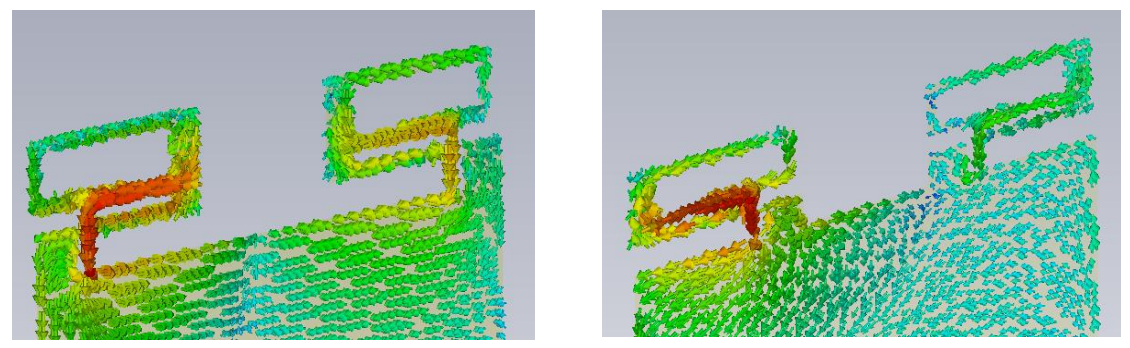

Figure 10. Diversity Antenna Surface Circuit Distribution at 2.45Ghz Type A(left) \& Type B(right) 

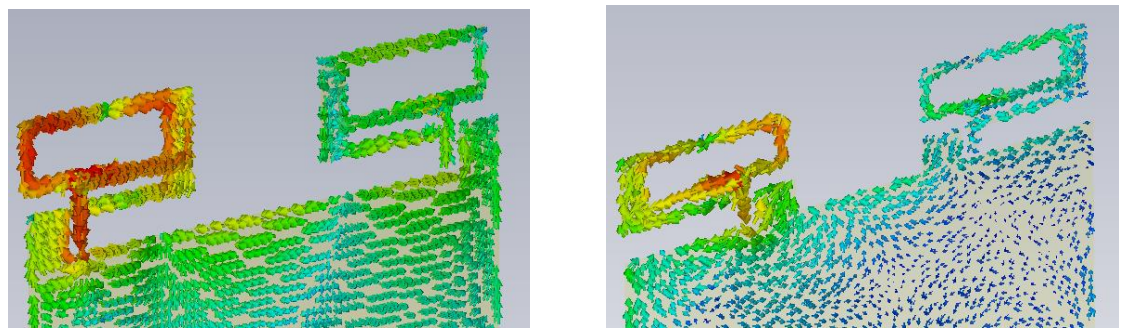

\section{Figure 11. Proposed Diversity Antenna Surface Circuit Distribution at 5.5Ghz Type A(left) \& Type B(right)}

\section{Experimental Result}

The proposed antenna is well fabricated and measured with an Agilent N5230C vector network analyzer and antenna measure system. Figure 12 shows the comparison results of measured and simulated return loss characteristics of the proposed antenna. The measured $-10 \mathrm{~dB}$ return loss listed as followed: $0.91-0.95 \mathrm{GHz}, 1.91-2.53 \mathrm{GHz}, 3.29-3.56 \mathrm{GHz}$ and 5.52-5.71GHz. The discrepancy between measured and simulated results is due to the fabrication and measurement deviation. The measured peak gain of the proposed antenna is shown in Figure 13. Measured peak gains of -2.8, 1.24, 5.13 and $4.04 \mathrm{dBi}$ and the antenna efficiency of $35 \%, 97 \%, 86 \%$ and $71 \%$ are achieved at $0.915,2.45,3.5$, and 5.7 $\mathrm{GHz}$, respectively.

The antenna lies on the xoz-plane as shown in the coordinate system. The measured and simulated radiation patterns in two principal planes, namely, E-plane (xz-plane, $\phi=$ 0 ) and H-plane (xy-plane, $\theta=90$ ), for $0.915,2.45,3.5$ and $5.7 \mathrm{GHz}$ are normalized and plotted in Figure 14-Figure 17, respectively. For the E-plane, a dumbbell-like radiation pattern is achieved which is similar to monopole radiation pattern, and for the $\mathrm{H}$-plane, nearly omnidirectional pattern is observed in basically all the frequency band. The above characteristics show that the antenna can provide stable gains in all the four bands.

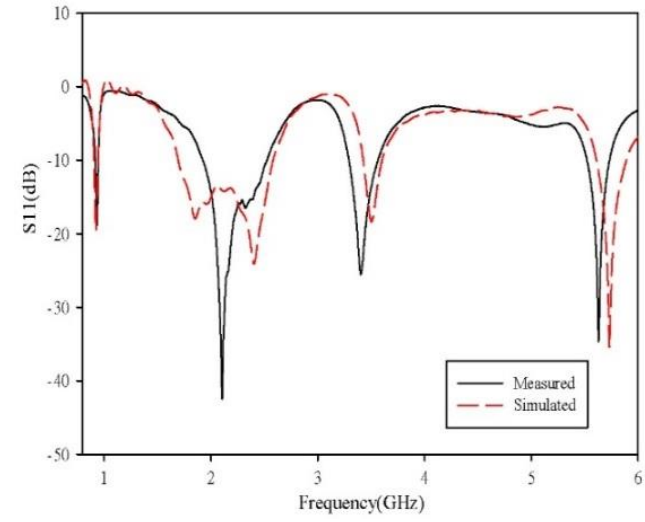

Figure 12. Measured and Simulated Return Loss Characteristic of Proposed Antenna

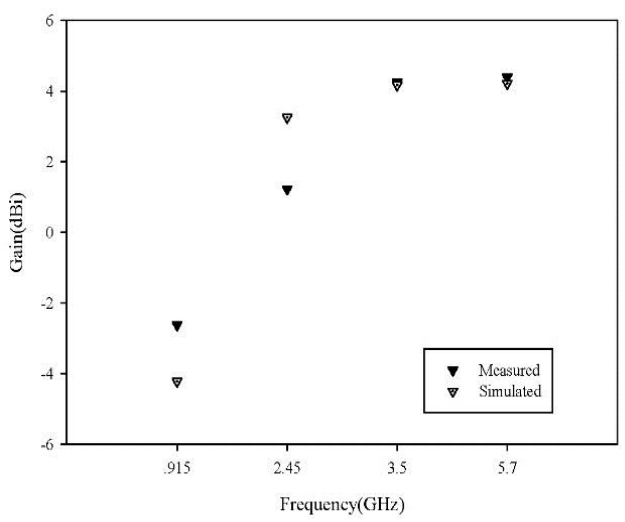

Figure 13. Measured Peak Gain of Proposed Antenna 


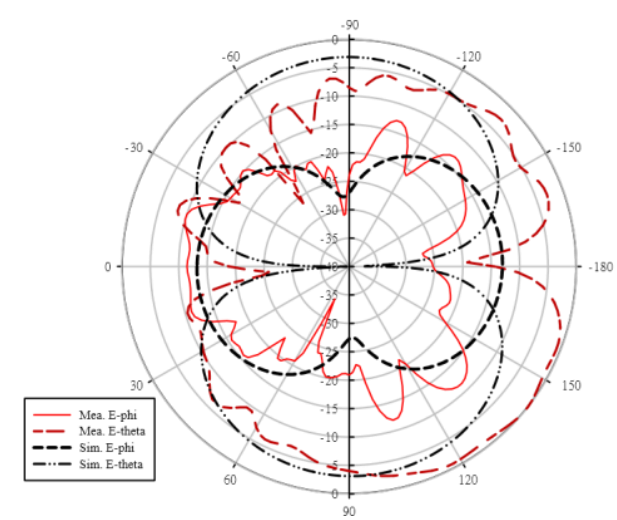

(a) E-plane

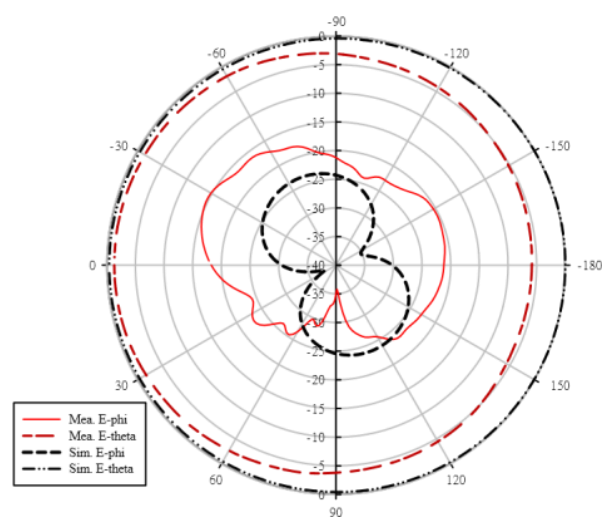

(b) H-plane

Figure 14. Simulated and Measured Radiation Pattern at $0.915 \mathrm{GHz}$

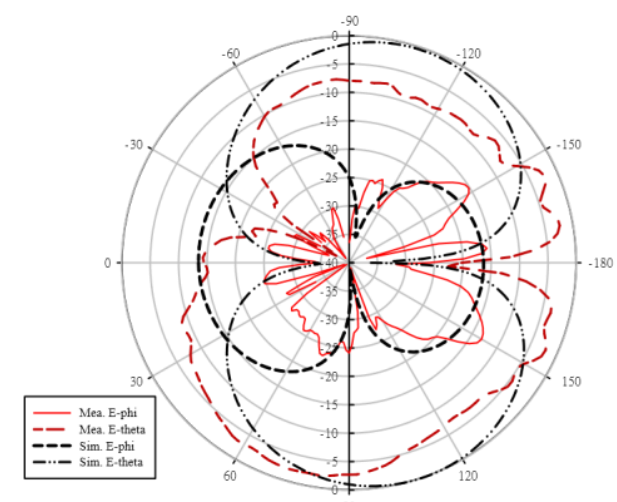

(a) E-plane

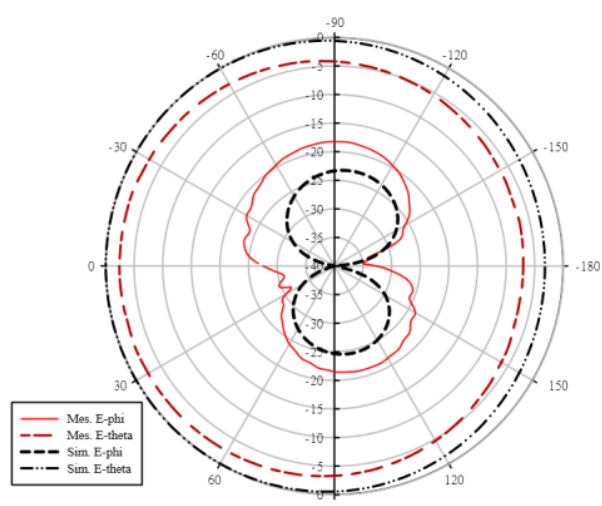

(b) H-plane

Figure 15. Simulated and Measured Radiation Pattern at $2.45 \mathrm{GHz}$

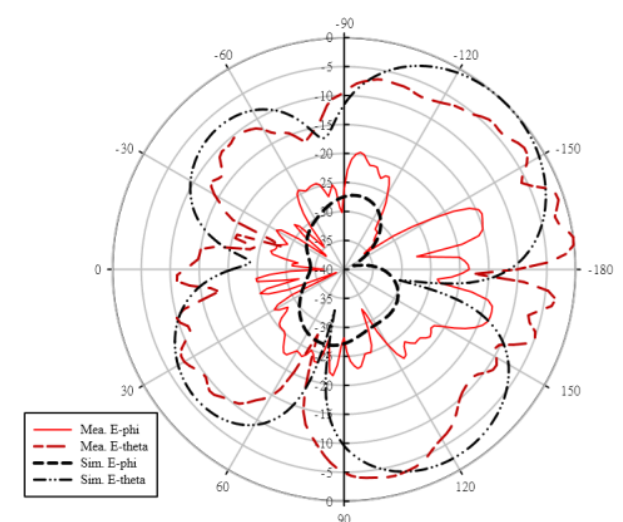

(a) E-plane

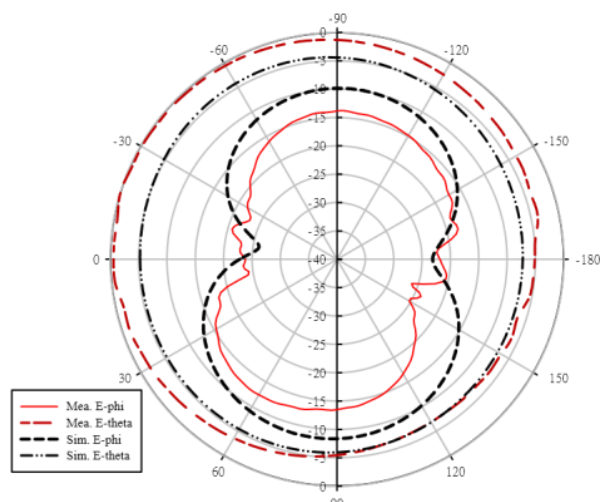

(b) H-plane

Figure 16. Simulated and Measured Radiation Pattern at $3.5 \mathrm{GHz}$ 


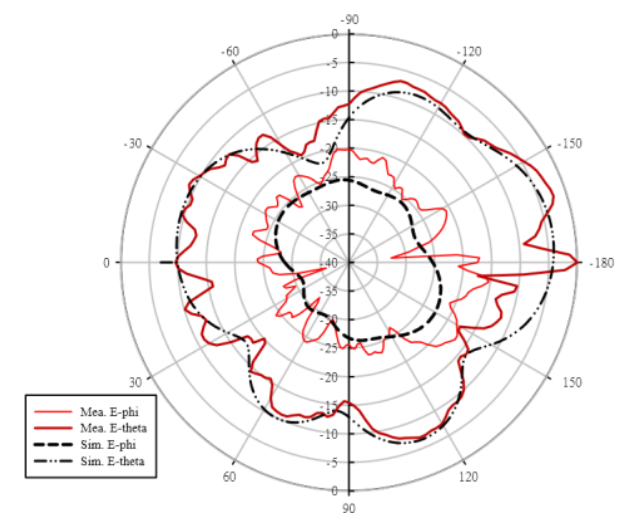

(a) E-plane

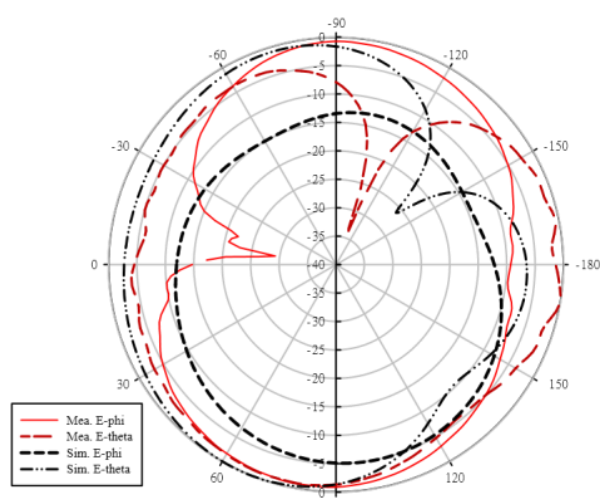

(b) H-plane

Figure 17. Simulated and Measured Radiation Pattern at $5.7 \mathrm{GHz}$

\section{Conclusion}

In this paper, a compact quad-band monopole diversity antenna for mobile handset devices is designed, fabricated and measured. Resonant modes are achieved at four desired frequencies $(0.915 / 2.45 / 3.5 / 5.5-5.8 \mathrm{GHz})$ with a compact size of $15.9 \times 25 \mathrm{~mm}^{2}$ of the radiation structure. Some diameter parameters in generating resonant frequencies and optimizing impedance matching are discussed in detail. The diversity performance and radiation mechanism is simulated and discussed by presenting the current distributions. Experimental results reveal the design has stable radiation properties, and adequate impedance bandwidths. Together with its compact volume, the proposed diversity antenna design is suitable for mobile handset device applications.

\section{Acknowledgment}

This work was supported in part by the National Natural Science Foundation of China by Grants (61170275), Beijing Higher Education Young Elite Teacher Project, and Beijing Key Laboratory of Work Safety Intelligent Monitoring.

\section{References}

[1] Kai Da Xu; Yong Hong Zhang; Spiegel, R.J.; Yong Fan; Joines, W.T.; Qing Huo Liu, "Design ofa StubLoaded Ring-Resonator Slot for Antenna Applications," Antennas and Propagation, IEEE Transactions on (2015), vol.63, no.2, pp.517,524.

[2] Sim, C.-Y.-D.; Yuan-Kai Shih; Ming-Hsuan Chang, "Compact slot antenna for wireless local areanetwork 2.4/5.2/5.8 GHz applications," Microwaves, Antennas \& Propagation, IET (2015), vol.9, no.6, pp.495,501.

[3] Insu Yeom; Jin Myung Kim; Chang Won Jung, "Dual-band slot-coupled patch antenna withbroad bandwidth and high directivity for WLAN access point," Electronics Letters(2014), vol.50, no.10, pp.726,728.

[4] H.-F. Huang and Y. Hu,"A Compact Dual-Band Printed Monopole Antenna for WiMax /WLANApplications” Progress In Electromagnetics Research Letters(2014), Vol. 49, 91-97.

[5] Mubarak S. Ellis, Zhi Qin Zhao, Jiang Niu Wu, Zai Ping Nie, and Qing-Huo Liu, "A New CompactMicrostrip-Fed Monopole Antenna for Triple Band WLAN/WiMAX Applications," Progress In Electromagnetics Research Letters(2014), Vol. 48, 129C135.

[6] M. Koohestani, A. A. Moreira, and A. K. Skrivervik, "A Novel Compact CPW-Fed PolarizationDiversity Ultrawideband Antenna," Antennas and Wireless Propagation Letters(2014), IEEE, vol. 13, pp. 563-566.

[7] Liu, L., et al.. "Compact MIMO Antenna for Portable Devices in UWB Applications." Antennas and Propagation, IEEE Transactions on(2013) PP(99): 1-1.

[8] Payandehjoo, K. and R. Abhari. "Compact Multi-Band PIFAs on a Semi-Populated Mobile Handset With Tunable Isolation." Antennas and Propagation, IEEE Transactions on (2013) 61(9): 4814-4819. 


\section{Authors}

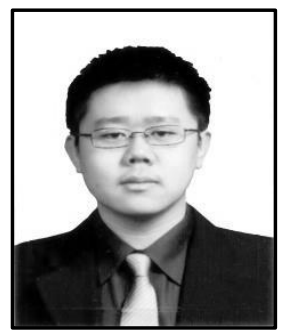

Yang Yang, was born in Jiangsu, China, in 1987. He received B.S. degrees in optical information science and technology form Beijing University of Posts and Telecommunications, Beijing, China, in 2009. He is currently working toward a Ph.D. degree in Electronic Science and Technology, Beijing University of Posts and Telecommunications. His research interests include diversity antenna, smart mobile device and Internet of Thing (IoT).

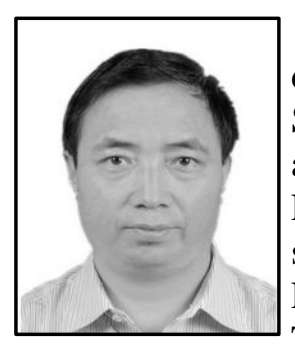

Yuan'An Liu, (M'93) received the B.E., M. Eng., and Ph.D. degrees in electrical engineering from the University of Electronic Science and Technology of China, Chengdu, China, in 1984, 1989, and 1992, respectively. In 1984, he joined the 26th Institute of Electronic Ministry of China to develop the inertia navigating system. In 1992, he began his first postdoctoral position in the EMC Laboratory, Beijing University of Posts and Telecommunications (BUPT), Beijing, China. In 1995, he started his second postdoctoral position in the Broadband Mobile Laboratory, Department of System and Computer Engineering, Carleton University, Ottawa, Canada. Since July 1997, he has been with the Wireless Communication Center, School of Telecommunication Engineering, BUPT, Beijing, China, as Professor, where he is involved in the development of nextgeneration cellular system, wireless LAN, Bluetooth application for data transmission, EMC design strategies for high-speed digital system, and EMI and EMS measuring sites with low cost and high performance. $\mathrm{He}$ is interested in smart antenna for high-capacity mobile, signal processing techniques in fading environments, EMC for high-speed digital system, ISI suppression, OFDM, and multicarrier system design. Dr. Liu is a Senior Member of the Electronic Institute of China.

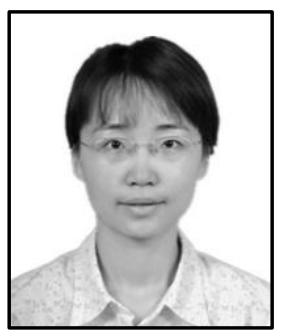

Fan Wu, Associate Professor, received the B.E. degree in electrical engineering from the University of Electronic Science and Technology of China, Chengdu, China, in 2004 and her Ph.D. degree in Beijing University of Posts and Telecommunications, Beijing, China, in 2009. Her research interests include Wireless Sensor Networks (WSNs), Internet of Thing (IoT) and Wireless Distributed Nerworks. 
International Journal of Future Generation Communication and Networking Vol. 9, No. 6 (2016) 